\title{
2-Cl-MGV-1 reduces neuronal apoptosis in the remote brain regions and ameliorates cognitive deficits after cortical infarct in hypertensive rats
}

Yicong Chen ${ }^{1}$, Leo Veenman², Fubing Ouyang ${ }^{1}$, Jiahui Liang ${ }^{1}$, Weixian Huang ${ }^{1}$, Moshe Gavish ${ }^{2}$, Jinsheng Zeng ${ }^{1^{*}}$

${ }^{1}$ Department of Neurology and Stroke Center, the First Affiliated Hospital, Sun Yat-sen University, Guangzhou, China

${ }^{2}$ Department of Neuroscience, Israel Institute of Technology, Haifa, Israel

\section{Objectives}

Focal cortical infarction causes neuronal apoptosis in the ipsilateral nonischemic thalamus and hippocampus, which is potentially associated with poststroke cognitive deficits. TSPO (translocator protein) is critical in regulating mitochondrial apoptosis pathways. We examined the effects of the novel TSPO ligand 2-Cl-MGV-1 on poststroke cognitive deficits, neuronal mitochondrial apoptosis, and secondary damage in the ipsilateral thalamus and hippocampus after cortical infarction.

\section{Methods}

One hundred fourteen hypertensive rats underwent successful distal middle cerebral artery occlusion $(n=76)$ or sham procedures $(n=38)$. 2-Cl-MGV-1 or dimethyl sulfoxide as vehicle was administrated 2 hours after distal middle cerebral artery occlusion and then for 6 or 13 days $(n=19$ per group). Spatial learning and memory were tested using the Morris water maze. Secondary degeneration and mitochondrial apoptosis in the thalamus and hippocampus were assessed using Nissl staining, immunohistochemistry, terminal deoxynucleotidyl transferase dUTP nick end labeling (TUNEL), JC-1 staining, and immunoblotting 7 and 14 days after surgery.

\section{Results}

2-Cl-MGV-1 administration significantly improved spatial cognitive impairment $(P<0.05)$ without amelioration of motor deficits after MCAO compared to vehicle. Infarct volumes did not significantly differ between the two groups at 7 or 14 days post-MCAO (both $P>0.05$ ). Increased TSPO expression was correlated to neuronal loss and microglia proliferation in the ipsilateral thalamus at 7 and 14 days after MCAO.

A
B

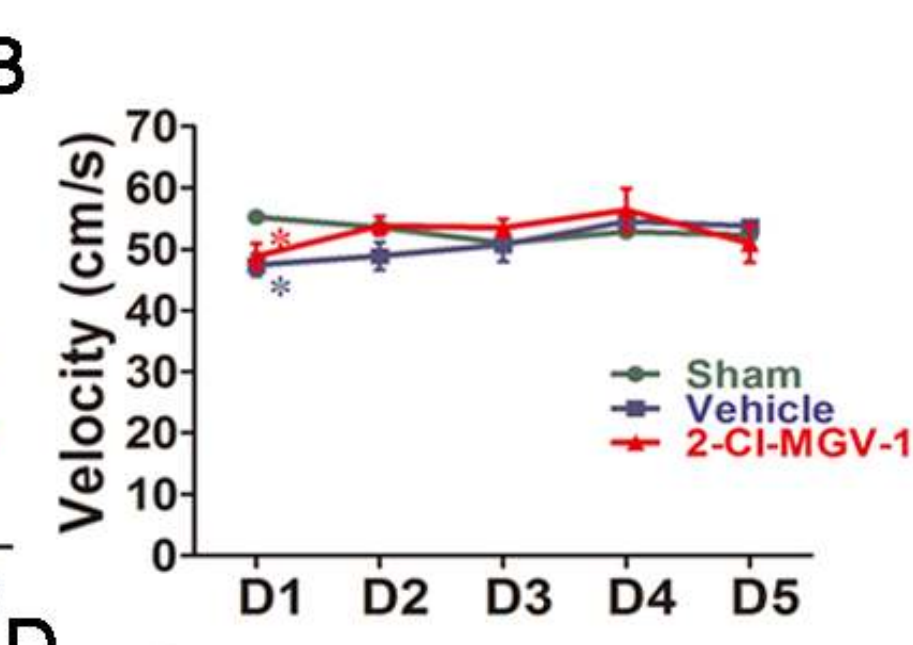

.

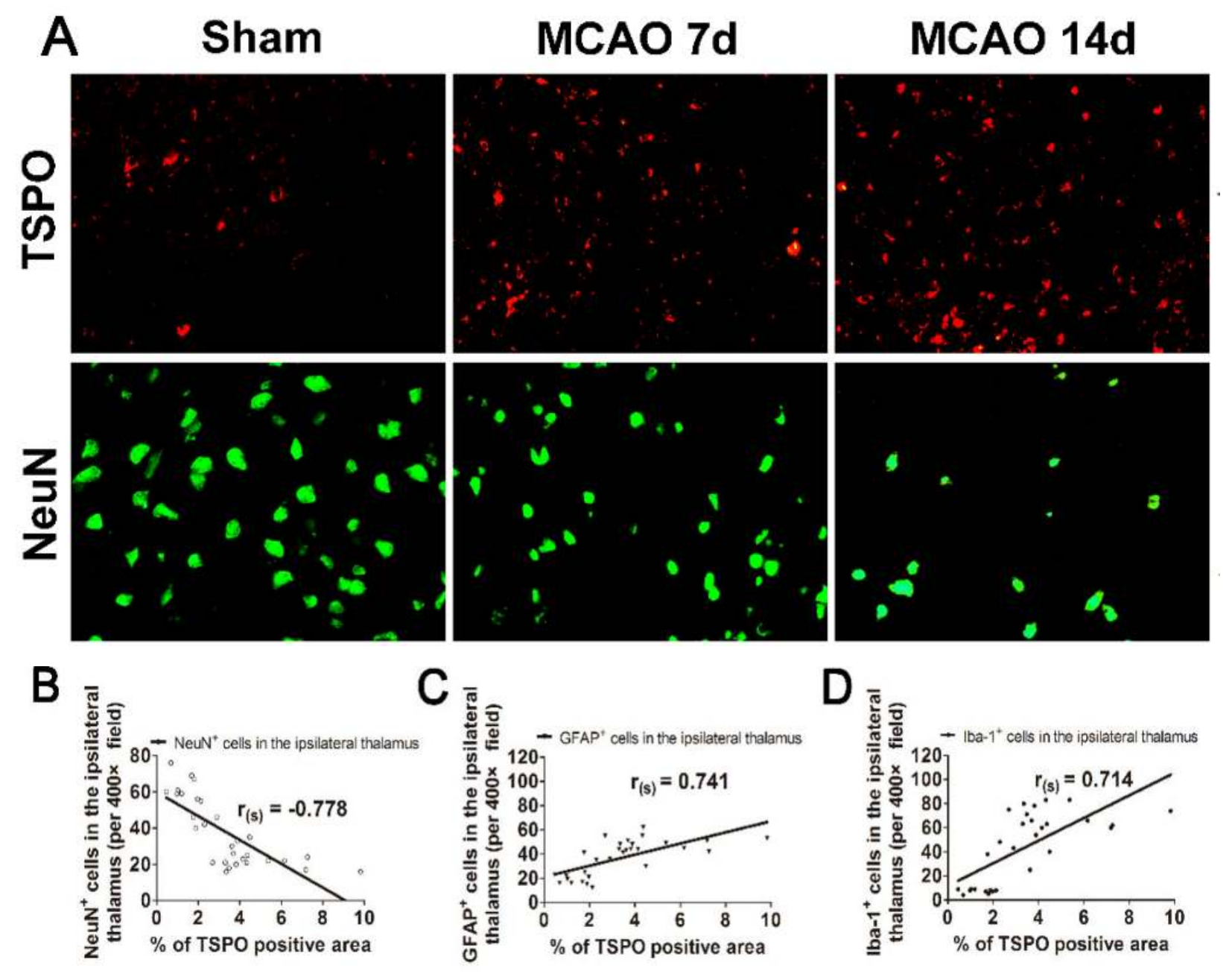

Figure 2. Increased expression of TSPO was correlated with neuronal loss and glia activation in the ipsilateral nonischemic thalamus after cortical infarction.
After 2-Cl-MGV-1 treatment, neuronal death and glial activation were remarkably decreased in the ipsilateral thalamic anterior nuclei and hippocampus post-MCAO $(P<0.05)$.

Figure 3. 2-Cl-MGV-1 ameliorated secondary Figure 4. 2-Cl-MGV-1 reduced nenronal damage in the ipsilateral anterior thalamic apoptosis in ipsilateral anterior thalamic nuclei after focal cerebral infarction.

nuclei after focal cerebral infarction.

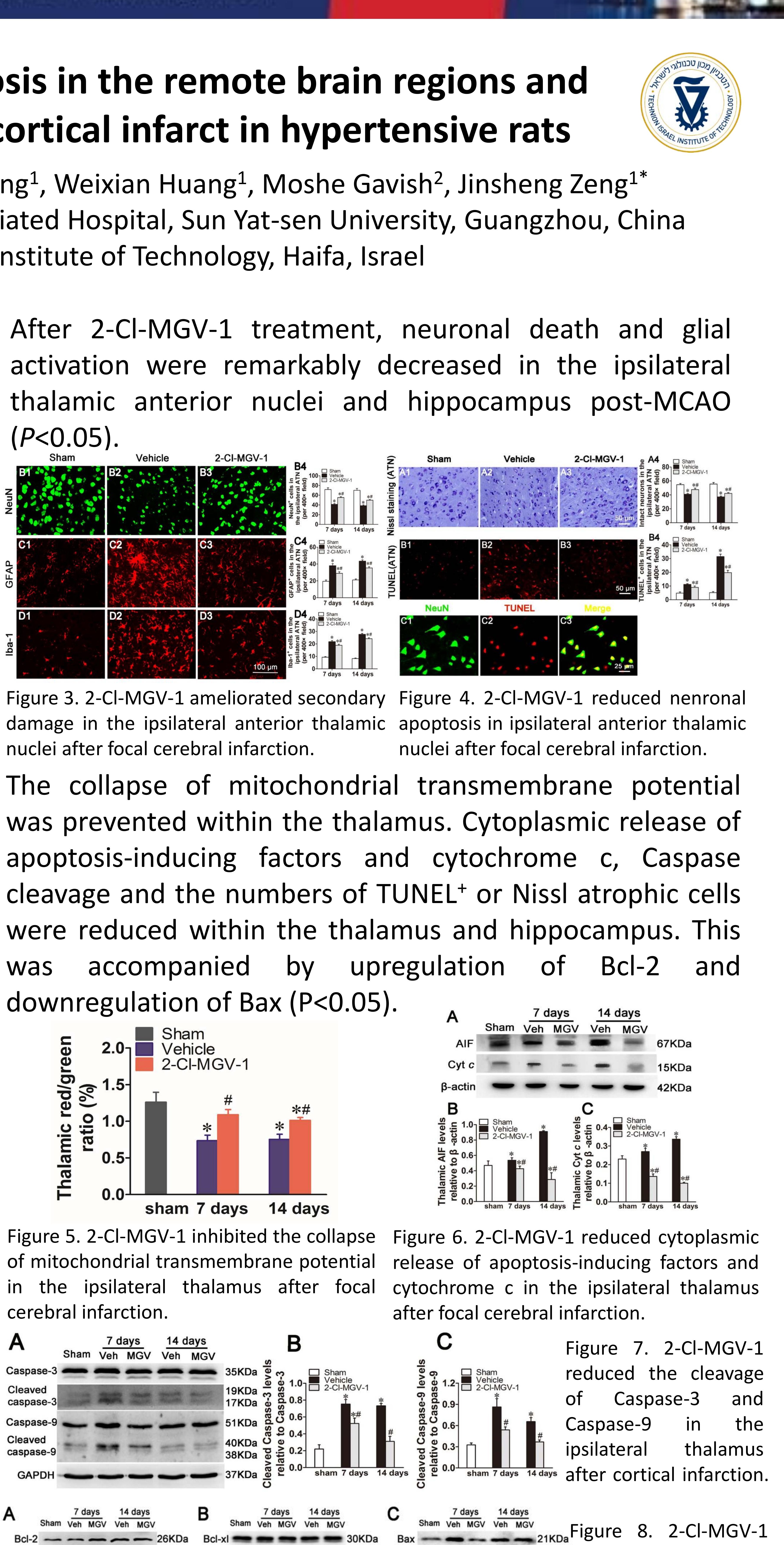

The collapse of mitochondrial transmembrane potential was prevented within the thalamus. Cytoplasmic release of apoptosis-inducing factors and cytochrome c, Caspase cleavage and the numbers of TUNEL ${ }^{+}$or Nissl atrophic cells were reduced within the thalamus and hippocampus. This was accompanied by upregulation of $\mathrm{Bcl}-2$ and downregulation of $\operatorname{Bax}(\mathrm{P}<0.05)$.
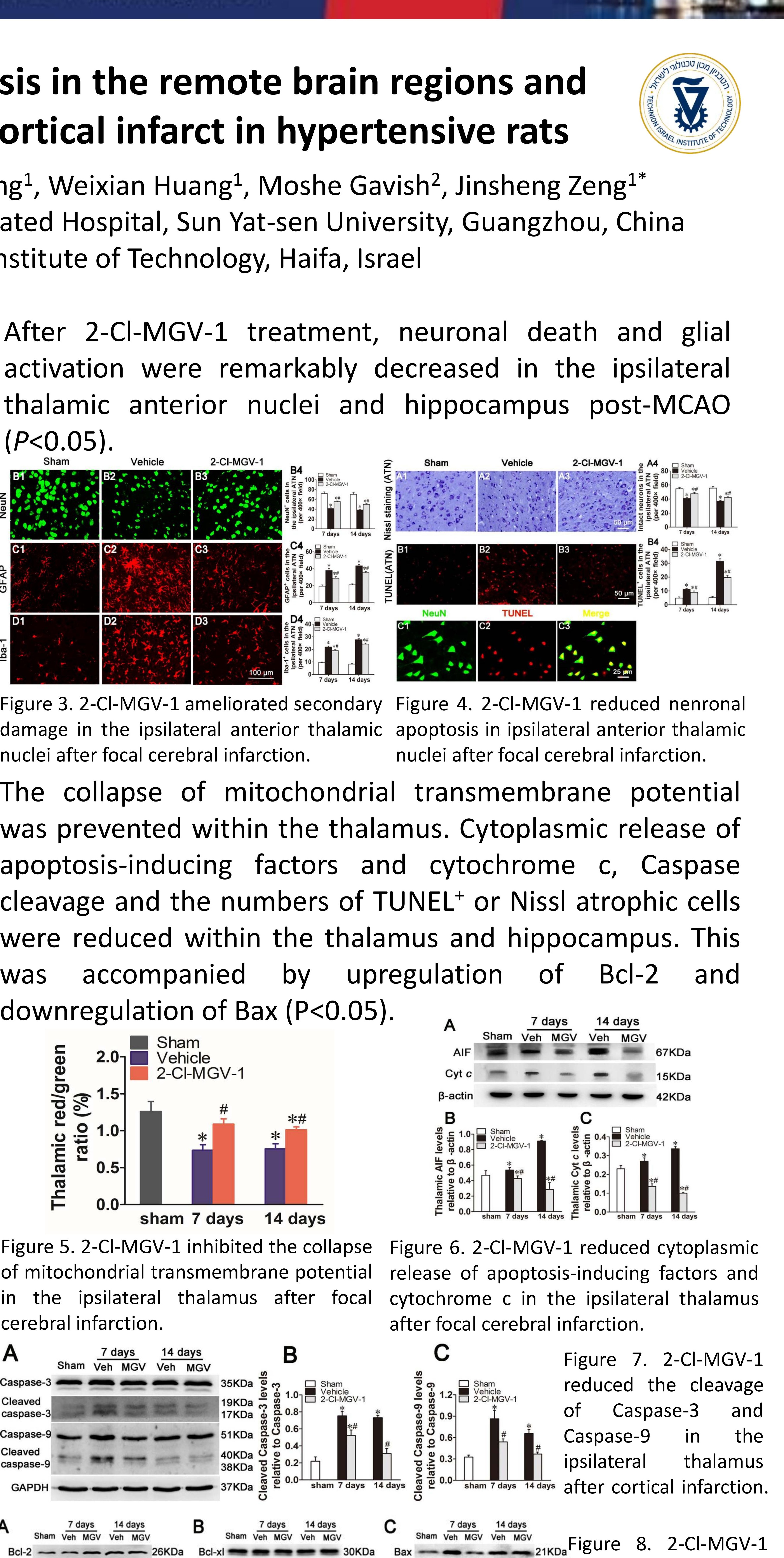

Figure 5. 2-Cl-MGV-1 inhibited the collapse Figure 6. 2-Cl-MGV-1 reduced cytoplasmic of mitochondrial transmembrane potential release of apoptosis-inducing factors and in the ipsilateral thalamus after focal cytochrome $c$ in the ipsilateral thalamus cerebral infarction.

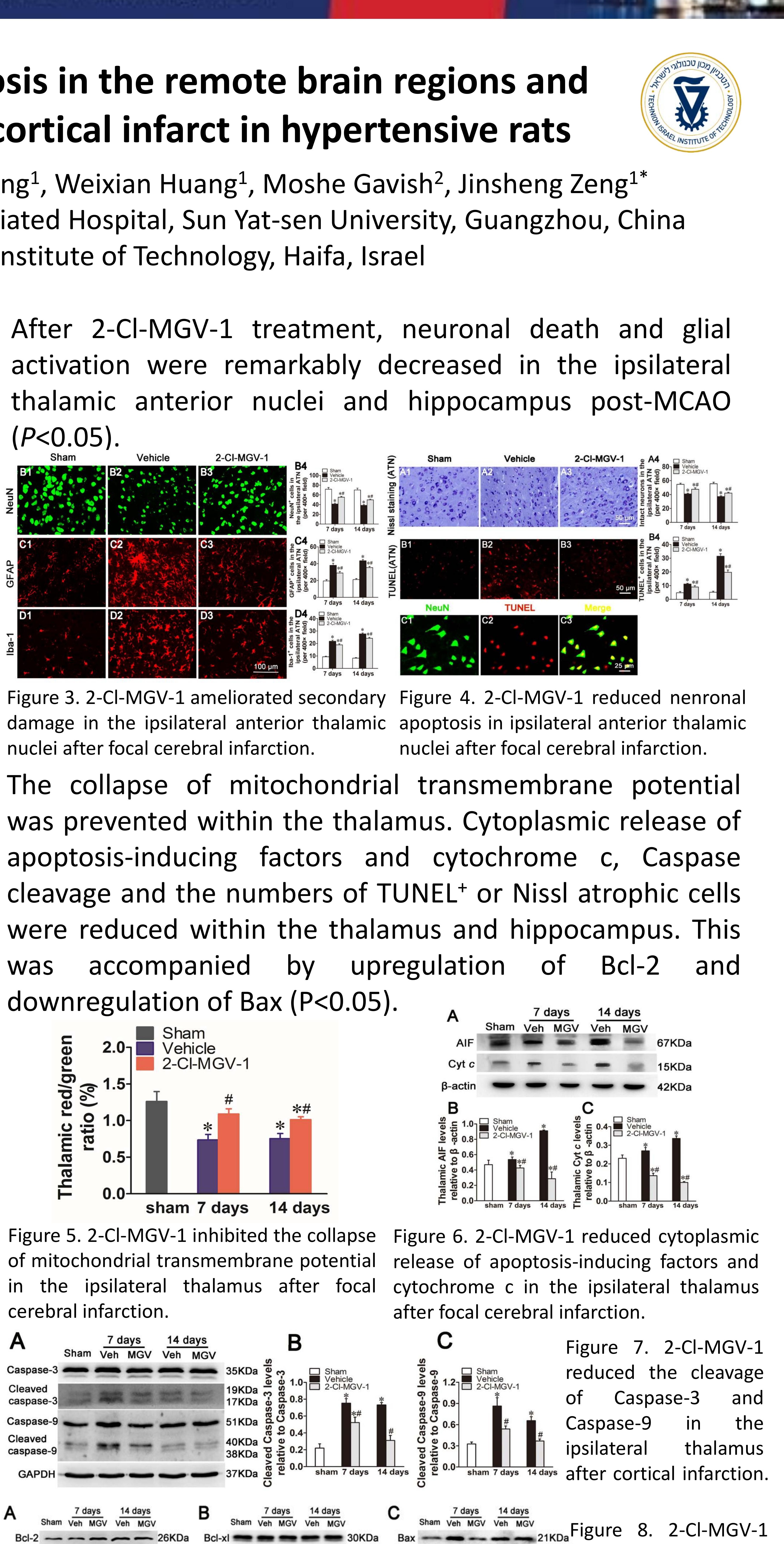
after focal cerebral infarction.
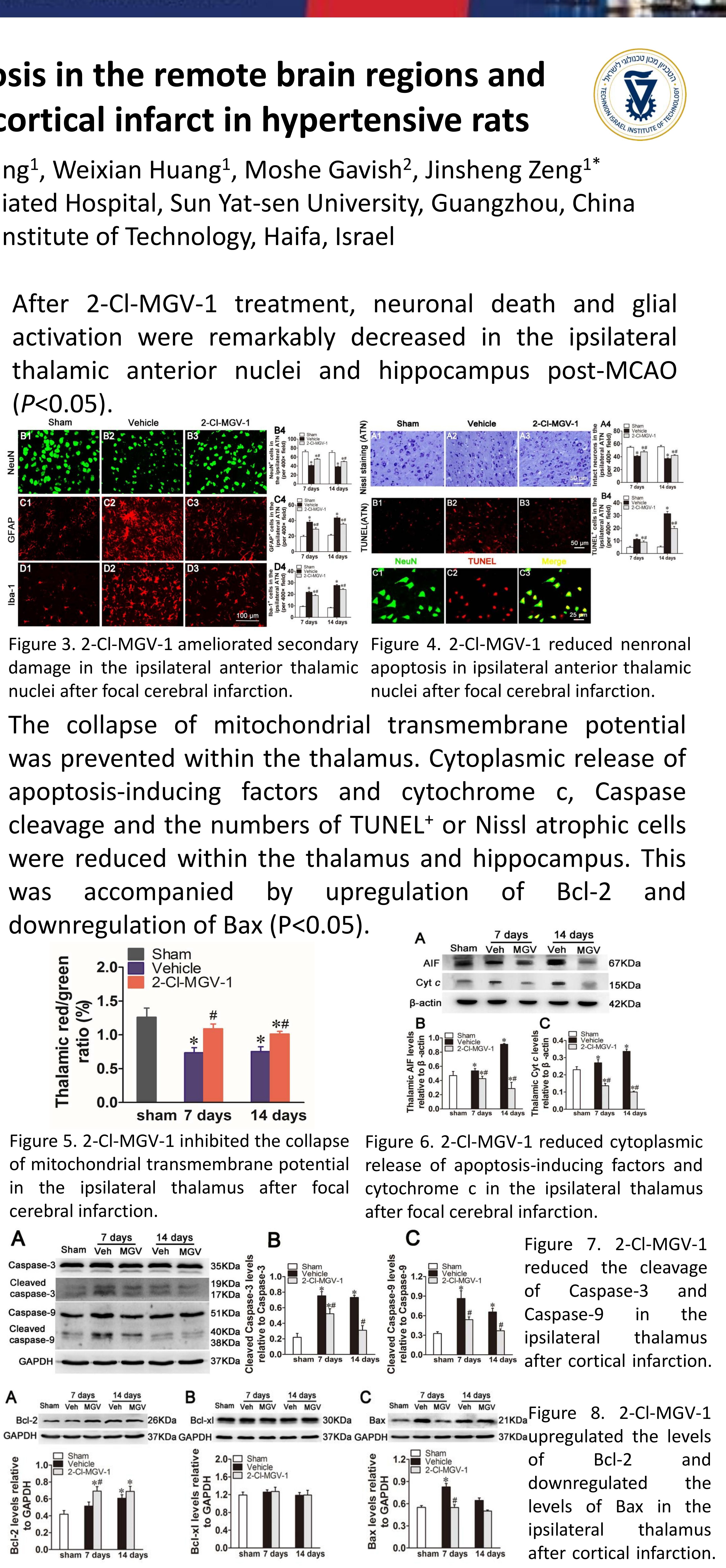

C Figure 7. 2-Cl-MGV-1

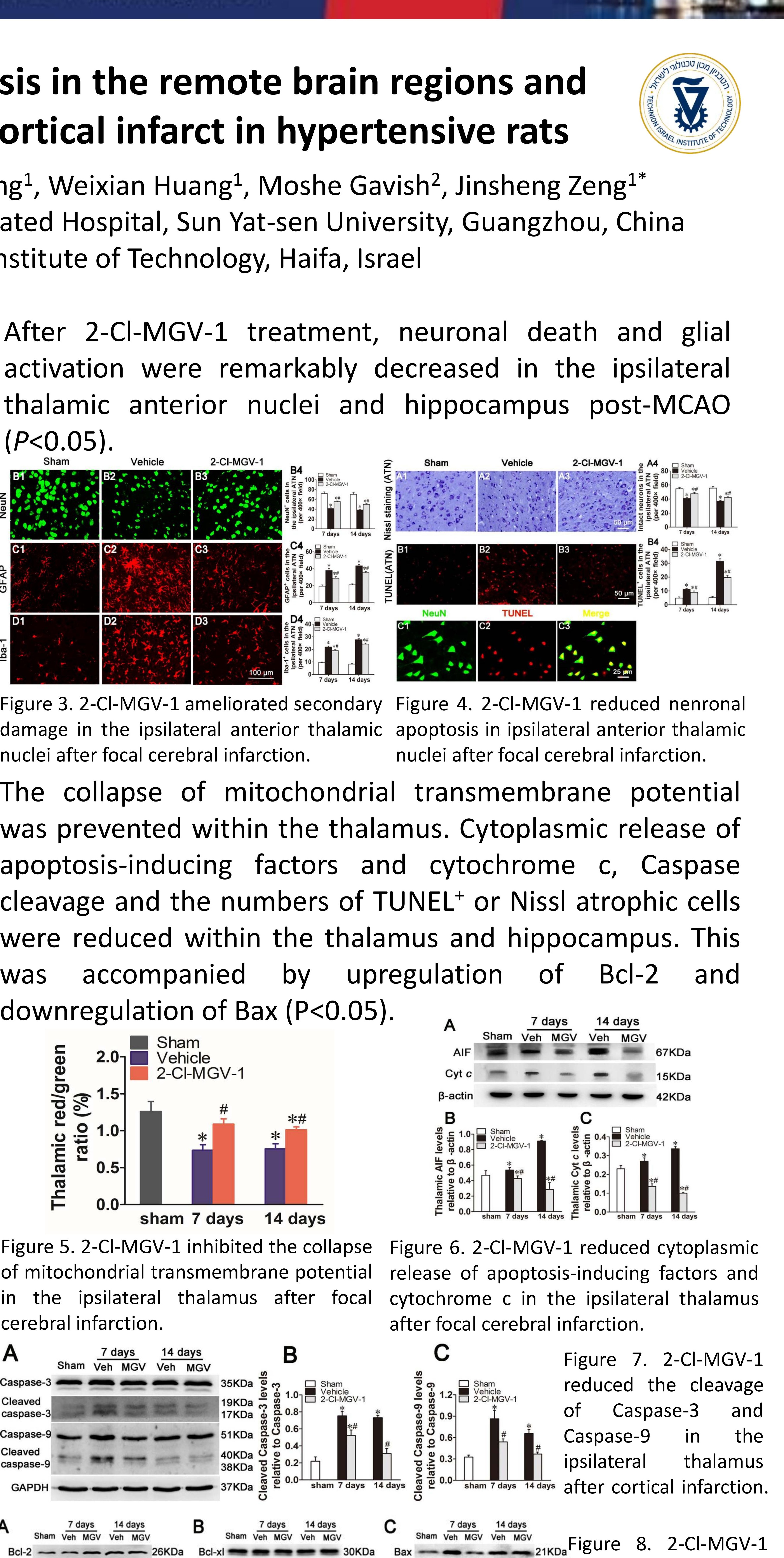
of Caspase-3 and ipsilateral thalamus after cortical infarction.

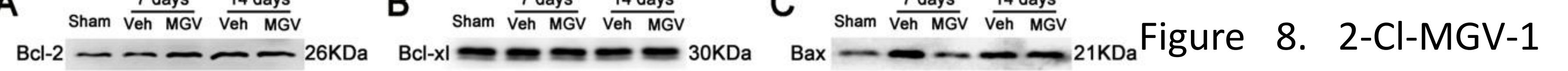

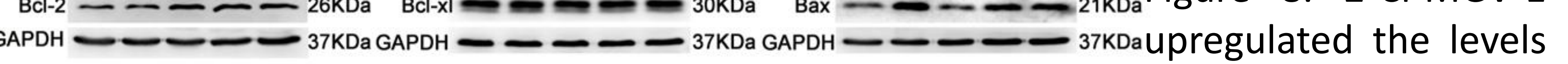

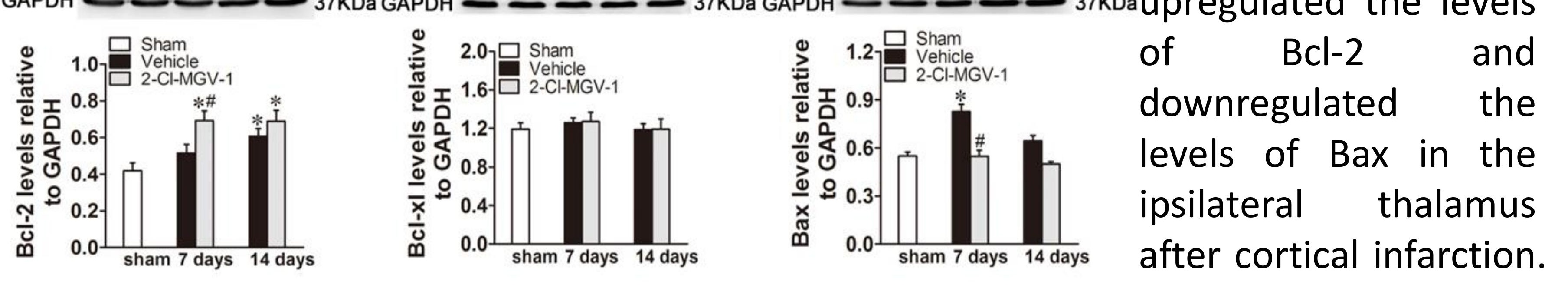

\section{Conclusions}

2-Cl-MGV-1 reduces neuronal apoptosis via mitochondrialdependent pathways and attenuates secondary damage in the non-ischemic thalamus and hippocampus, potentially contributing to ameliorated cognitive deficits after cortical infarction. 